\title{
A Simple Tableau System for the Logic of Elsewhere $^{\star}$
}

\author{
Stéphane Demri \\ Leibniz-Imag \\ 46, Avenue Félix Viallet, \\ 38031 Grenoble Cedex, France \\ e-mail: stephane.demri@imag.fr
}

\begin{abstract}
We analyze different features related to the mechanization of von Wright's logic of elsewhere $\mathcal{E}$. First, we give a new proof of the NP-completeness of the satisfiability problem (giving a new bound for the size of models of the satisfiable formulae) and we show that this problem becomes linear-time when the number of propositional variables is bounded. Although $\mathcal{E}$ and the well-known propositional modal S5 share numerous common features we show that $\mathcal{E}$ is strictly more expressive than S5 (in a sense to be specified). Second, we present a prefixed tableau system for $\mathcal{E}$ and we prove both its soundness and completeness. Two extensions of this system are also defined, one related to the logical consequence relation and the other related to the addition of modal operators (without increasing the expressive power). An example of tableau proof is also presented. Different continuations of this work are proposed, one of them being to implement the defined tableau system, another one being to extend this system to richer logics that can be found in the literature.
\end{abstract}

\section{Introduction}

In $[\text { Seg81 }]^{1} \mathrm{~K}$. Segerberg gives a proof for a complete axiomatization of the logic of elsewhere defined by von Wright (herein called $\mathcal{E}$ ). The language of $\mathcal{E}$ contains a modal operator $[\neq]$ related to the complement of the diagonal relation in the Kripke-style semantics. Enriched languages of classical modal logics have been the object of very active research (see e.g. [GPT87,Gor90,PT91,Rij92,GG93]) which confirms Segerberg's opinion in [Seg81] about the scope of von Wright's logic of elsewhere. Actually very sophisticated machineries exist to study the expressive power of such logics. The main motivation of our paper is to analyze some computational issues about the mechanization of $\mathcal{E}$. As far as we know, the construction of proof systems for modal logics with enriched languages has been neglected in the past although tableau systems have been defined for various modal logics (see e.g. [Fit88,Cat91,Gor92,Mas94,Ogn94]). There exist of course

\footnotetext{
* This work has been supported by the Centre National de la Recherche Scientifique (C.N.R.S.). Paper to be presented at Tableaux'96.

1 The author thanks Prof. Ewa Orłowska for pointing him this work.
} 
Hilbert-style proof systems but neither resolution nor tableau proof system exist for these logics. This lack is quite surprising when considering the numerous recent works related to the mechanization of modal logics in the large sense of the word (see e.g. recently [OSH95,Non95]). Using the methodology developed in [Fit83], we define a sound and complete prefixed tableau system for $\mathcal{E}$. This system can be easily implemented in any existing tableau prover.

The structure of the paper is the following. In Section 2, we recall the features of the logic of elsewhere $\mathcal{E}$ as well as Segerberg's complete axiomatization. In section 3 , we give a new proof of the $\mathbf{N P}$-completeness of the satisfiability problem for $\mathcal{E}$ and we show that when the set of propositional variables is bounded, this problem is linear in time. In Section 4 , we show that $\mathcal{E}$ and S5 have different expressive powers although they share numerous common features. In Section 5 we define a prefixed tableau system and we prove its soundness and completeness with respect to the semantics of $\mathcal{E}$. As a side-effect, another proof of the decidability of the satisfiability problem for $\mathcal{E}$ is given. In Section 6 we extend our initial tableau in two directions: one by considering the notion of logical consequence and the other by extending the language of $\mathcal{E}$. In the latter case, the expressivity of the extension is the same as in the initial language but the additional modal operators are quite natural. We conclude the paper by presenting an example of tableau proof and we discuss possible extensions of this work.

\section{Logic of Elsewhere}

The language of the logic of elsewhere $\mathcal{E}$, written $\mathrm{L}$, is determined by three sets which are supposed to be pairwise disjoint, a set $\phi_{0}=\{P, Q, \ldots\}$ of propositional variables, the set $\{\neg, \wedge\}$ of propositional operators and the set $\{[\neq],\langle\neq\rangle\}$ of modal operators. The set of formulae $\operatorname{FOR}\left(\phi_{0}\right)$ is the smallest set that satisfies the conditions: (1) $\phi_{0} \subseteq \operatorname{FOR}\left(\phi_{0}\right),(2)$ if $\oplus$ is any $n$-ary propositional operator and $A_{1}, \ldots, A_{n} \in \operatorname{FOR}\left(\phi_{0}\right)$ then $\oplus\left(A_{1}, \ldots, A_{n}\right) \in \operatorname{FOR}\left(\phi_{0}\right)$ and $(3)$ if $A \in \operatorname{FoR}\left(\phi_{0}\right)$ then $\{[\neq] A,\langle\neq\rangle A\} \subseteq \operatorname{FOR}\left(\phi_{0}\right)$. The operators $\vee, \Rightarrow, \Leftrightarrow$ are used as abbreviations with their standard meaning. We write $\operatorname{sub}(A)$ (resp. $m w(A))$ to denote the set of subformulae of the formula $A$ (resp. the modal weight of $A$, i.e. the number of occurrences of modal operators in $A$ ). The length of a formula $A$, written $|A|$, is the number of symbols occurring in $A$. We briefly recall the Kripke-style semantics for the logic of elsewhere.

Definition 1. A model for $\mathcal{E}$ (also called $\mathcal{E}$-model) $\mathcal{M}$ is a pair $(W, V)$ where, $W$ is a non-empty set of worlds and $V$ is a mapping $\phi_{0} \rightarrow \mathcal{P}(W)$, the power set of $W$.

Given any model $\mathcal{M}$, any world $w \in W$, and any formula $A \in \operatorname{FOR}\left(\phi_{0}\right)$, the expression " $w$ satisfies $A$ in $\mathcal{M}$ " $(\mathcal{M}, w \models A)$ is defined as follows:

$-\mathcal{M}, w \models P$ iff $w \in V(P)$ for all $P \in \phi_{0}$,

$-\mathcal{M}, w \models \neg A$ iff not $\mathcal{M}, w \models A$, 
- $\mathcal{M}, w \models A_{1} \wedge A_{2}$ iff $\mathcal{M}, w \models A_{1}$ and $\mathcal{M}, w \models A_{2}$,

- $\mathcal{M}, w \models\langle\neq\rangle A$ iff there is $w^{\prime} \in W \backslash\{w\}$ such that $\mathcal{M}, w^{\prime} \models A$,

$-\mathcal{M}, w \models[\neq] A$ iff for all $w^{\prime} \in W \backslash\{w\}, \mathcal{M}, w^{\prime} \models A$.

The classical logical connectives behave as usually. A formula $A \in \operatorname{FOR}\left(\phi_{0}\right)$ is said to be satisfiable (for $\mathcal{E}$ ) if there is a $\mathcal{E}$-model $\mathcal{M}$ and a world $w$ of $\mathcal{M}$ such that $\mathcal{M}, w \models A$. A formula $A \in \operatorname{FOR}\left(\phi_{0}\right)$ is said to be valid in a model $\mathcal{M}$ iff for all $w \in W, \mathcal{M}, w \models A$. A formula $A \in \operatorname{FOR}\left(\phi_{0}\right)$ is said to be valid iff it is valid in every $\mathcal{E}$-model. For any set $S \subseteq \mathcal{P}\left(\operatorname{FOR}\left(\phi_{0}\right)\right), A \in \operatorname{FOR}\left(\phi_{0}\right)$, we write $S \models A$ iff for all models $\mathcal{M}$ such that for every $B \in S, B$ is valid in $\mathcal{M}$, we have $A$ is valid in $\mathcal{M}$.

The universal modal operator $\mathcal{U}$ (see e.g. [GP92]) can be defined by $\mathcal{U} A \equiv A \wedge$ $[\neq] A$. Moreover the operator $\langle!\rangle$ defined by $\langle!\rangle A \equiv(A \wedge[\neq] \neg A) \vee\langle\neq\rangle(A \wedge[\neq] \neg A)$ expresses that a property is satisfied in a unique world of the model. Numerous conditions can be expressed in L with respect to the notion of modal definability $(\operatorname{card}(W) \leq n, \operatorname{card}(W)>m, \ldots)$. For any set $S, \operatorname{card}(S)$ denotes the cardinal of $S$.

The Hilbert-style system DL contains the following set of axioms

P. all formulae having the form of a classical propositional tautology

K. $[\neq](P \Rightarrow Q) \Rightarrow([\neq] P \Rightarrow[\neq] Q)$

S. $P \Rightarrow[\neq]\langle\neq\rangle P$

A. $\langle\neq\rangle\langle\neq\rangle P \Rightarrow(P \vee\langle\neq\rangle P)$

and the rules of inference

MP. from $A$ and $A \Rightarrow B$ infer $B$

NR. from $A$ infer $[\neq] A$.

Proposition 1. [Rij92] Let $S \cup\{A\} \subseteq \operatorname{FOR}\left(\phi_{0}\right)$. Then $S \vdash_{\mathrm{DL}} A$ iff $S \models A$.

The system DL corresponds to the axiomatization of $\mathcal{E}$ defined in [Seg81].

\section{Complexity of the Satisfiability Problem}

The satisfiability problem for $\mathcal{E}$ is decidable and can be proved to be NPcomplete (see [Rij92]). We give below an original proof of the NP-completeness of the satisfiability problem and we propose a new bound for the size of the models for the satisfiable formulae. The proof of Proposition 2 follows the lines of the proof of Lemma 6.1 in [Lad77] although adequate modifications are made.

Proposition 2. A formula $A \in \operatorname{FOR}\left(\phi_{0}\right)$ is satisfiable iff it is satisfiable in a $\mathcal{E}$-model $(W, V)$ such that $\operatorname{card}(W) \leq 2 \times m w(A)+1$.

Proof. Assume there is $\mathcal{M}=(W, V)$ a model, $u \in W$ such that $\mathcal{M}, u \models A$. Since $[\neq] P \Leftrightarrow \neg\langle\neq\rangle \neg P$ is valid, we can assume without loss of generality that $[\neq]$ does not occur in $A$ (replacing in $A$ the occurrences of $[\neq]$ by $\neg\langle\neq\rangle \neg$ does not change 
the modal weight). Let $\langle\neq\rangle^{A}$ be the set $\{\langle\neq\rangle B:\langle\neq\rangle B \in \operatorname{sub}(A), \mathcal{M}, u \models\langle\neq\rangle B\}$. For each $\langle\neq\rangle B \in\langle\neq\rangle^{A}$, we write $S_{B}$ to denote the set $\left\{u^{B} \in W \backslash\{u\}: \mathcal{M}, u^{B} \models\right.$ $B\}$ and we define $S_{B}^{1,2} \subseteq S_{B}$ such that if $\operatorname{card}\left(S_{B}\right)=1$ then $S_{B}^{1,2}=S_{B}$ otherwise $\operatorname{card}\left(S_{B}^{1,2}\right)=2$ (the elements of $S_{B}^{1,2}$ can be viewed as representative elements of $\left.S_{B}\right)$. Let $\mathcal{M}^{\prime}=\left(S^{\prime}, V^{\prime}\right)$ be the $\mathcal{E}$-model such that

$-S^{\prime}=\bigcup\left\{S_{B}^{1,2}:\langle\neq\rangle B \in\langle\neq\rangle^{A}\right\} \cup\{u\}$,

- $V^{\prime}$ is the restriction of $V$ to $S^{\prime}$

Observe that $\operatorname{card}\left(S^{\prime}\right) \leq 2 \times m w(A)+1$ since $\operatorname{card}\left(\langle\neq\rangle^{A}\right) \leq m w(A)$. We shall show that for all $u^{\prime} \in S^{\prime}$ and for all $B \in \operatorname{sub}(A) \mathcal{M}, u^{\prime} \models B$ iff $\mathcal{M}^{\prime}, u^{\prime} \models B$. We proceed by induction on the structure of $B$. The only nontrivial case is when $B$ is of the form $\langle\neq\rangle B^{\prime}$. Assume $\mathcal{M}, u^{\prime} \models\langle\neq\rangle B^{\prime}$. If $u^{\prime}=u$ then there is $u^{B^{\prime}} \in$ $S^{\prime} \backslash\{u\}$ such that $\mathcal{M}, u^{B^{\prime}} \models B^{\prime}$. By the induction hypothesis, $\mathcal{M}^{\prime}, u^{B^{\prime}} \models B^{\prime}$ and therefore $\mathcal{M}^{\prime}, u^{\prime} \models\langle\neq\rangle B^{\prime}$. If $u^{\prime} \neq u$ then there is $u^{\prime \prime} \in W \backslash\left\{u^{\prime}\right\}$ such that $\mathcal{M}, u^{\prime \prime} \models B^{\prime}$. If $u=u^{\prime \prime}$ then by the induction hypothesis, $\mathcal{M}^{\prime}, u \models B^{\prime}$ and therefore $\mathcal{M}^{\prime}, u^{\prime} \models\langle\neq\rangle B^{\prime}$. Now consider the case $u^{\prime \prime} \neq u$. If $u^{\prime \prime} \in S_{B^{\prime}}^{1,2}$ then $u^{\prime \prime} \in S^{\prime}$ and therefore by the induction hypothesis, $\mathcal{M}^{\prime}, u^{\prime \prime} \models B^{\prime}$. Hence $\mathcal{M}^{\prime}, u^{\prime} \models\langle\neq\rangle B^{\prime}$. If $u^{\prime \prime} \notin S_{B^{\prime}}^{1,2}$ then $\operatorname{card}\left(S_{B^{\prime}}^{1,2}\right)=2$. So there is $u^{*} \in S_{B^{\prime}}^{1,2} \backslash\left\{u^{\prime}\right\}$ such that $\mathcal{M}, u^{*} \models B^{\prime}$. By the induction hypothesis, $\mathcal{M}^{\prime}, u^{*} \models B^{\prime}$ and therefore $\mathcal{M}^{\prime}, u^{\prime} \models\langle\neq\rangle B^{\prime}$.

Now assume $\mathcal{M}, u^{\prime} \not \forall\langle\neq\rangle B^{\prime}$. So for all $u^{\prime \prime} \in W \backslash\left\{u^{\prime}\right\} \mathcal{M}, u^{\prime \prime} \not \models B^{\prime}$. In particular for all $u^{\prime \prime} \in\left(S^{\prime} \backslash\left\{u^{\prime}\right\}\right) \subseteq\left(W \backslash\left\{u^{\prime}\right\}\right) \mathcal{M}, u^{\prime \prime} \not \models B^{\prime}$. By the induction hypothesis, for all $u^{\prime \prime} \in\left(S^{\prime} \backslash\left\{u^{\prime}\right\}\right) \mathcal{M}^{\prime}, u^{\prime \prime} \not \neq B^{\prime}$ and therefore $\mathcal{M}^{\prime}, u^{\prime} \not \neq\langle\neq\rangle B^{\prime}$.

In [Rij92], it is stated that it has been proved in [DSVEB90] that any satisfiable formula $A$ has a model with a most $4 \times|A|$ worlds. Proposition 2 provides an alternative bound for the size of the models for the satisfiable formulae.

Proposition 3. The satisfiability problem is NP-complete.

Proof. As classical propositional calculus is part of $\mathcal{E}$, Cook's Theorem ([Coo71]) implies that deciding satisfiability is NP-hard. By Proposition 2, $A$ is satisfiable iff there is a model $\mathcal{M}=(W, V)$ with $\operatorname{card}(W) \leq 2 \times m w(A)+1$ and $u \in W$ such that $\mathcal{M}, u \models A$. Such a model can be "guessed" nondeterministically and checked in polynomial time.

In the rest of the section we assume that $\phi_{0}$ is a finite set of propositional variables. Such a limitation is sometimes reasonable for particular applications. We shall show that the satisfiability problem for $\mathcal{E}$ becomes linear-time. We follow the methodology presented in [Hal95] although significant variations are operated.

Proposition 4. There is a natural number $K$ such that for any model $\mathcal{M}=$ $(W, V)$ with $W$ finite and for any formula $A \in \operatorname{FOR}\left(\phi_{0}\right)$, there is an algorithm for checking whether there is $w \in W$ such that $\mathcal{M}, w \models A$ that runs in times at most $K \times \operatorname{card}(W) \times|A|$. 
Proof. Similar to the proof of Proposition 3.1 in [HM92].

For each model $\mathcal{M}=(W, V)$ we define the equivalence relation $\equiv_{\mathcal{M}}$ such that $u \equiv_{\mathcal{M}} v$ iff for all $P \in \phi_{0}, u \in V(P)$ iff $v \in V(P)$. We write $|u|_{V}$ to denote the equivalence classes with respect to $\equiv_{\mathcal{M}}$. Observe that for all $A \in \operatorname{FOR}\left(\phi_{0}\right)$, if $|u|_{V}=|v|_{V}$ then $\mathcal{M}, u \models A$ iff $\mathcal{M}, v \models A$.

Proposition 5. Let $\mathcal{M}=(W, V)$ be a model and $u, u^{\prime} \in W$ such that $|u|_{V}=$ $\left|u^{\prime}\right|_{V}$. Take any $u^{\prime \prime} \notin W$ and define the model $\mathcal{M}^{\prime}=\left(W \cup\left\{u^{\prime \prime}\right\}, V^{\prime}\right)$ such that for all $P \in \phi_{0}, V^{\prime}(P)=V(P)$ if $u \notin V(P)$ otherwise $V^{\prime}(P)=V(P) \cup\left\{u^{\prime \prime}\right\}$. Then for all $A \in \operatorname{FOR}\left(\phi_{0}\right), v \in W, \mathcal{M}, v \models A$ iff $\mathcal{M}^{\prime}, v \models A$.

Proof. The worlds $u^{\prime}$ and $u^{\prime \prime}$ can be seen as copies of the world $u$. The proof is by induction on the length of $A$. By way of example we only show that if $\mathcal{M}, v \models[\neq] B$ then $\mathcal{M}^{\prime}, v \models[\neq] B$. So assume $\mathcal{M}, v \models[\neq] B$. If $v \in\left\{u, u^{\prime}\right\}$ then it follows that $\mathcal{M}, v \models B$. So for all $w \in W, \mathcal{M}, w \models B$. By the induction hypothesis, for all $w \in W, \mathcal{M}^{\prime}, w \models B$. Since $\mathcal{M}^{\prime}, v \models B$ iff $\mathcal{M}^{\prime}, u^{\prime \prime} \models B$ (remember $\left|u^{\prime \prime}\right|_{V^{\prime}}=|v|_{V^{\prime}}$ ), for all $w \in W^{\prime}, \mathcal{M}^{\prime}, w \models B$ and therefore $\mathcal{M}^{\prime}, v \models$ $[\neq] B$. Now assume $v \notin\left\{u, u^{\prime}\right\}$. For all $w \in W \backslash\{v\}, \mathcal{M}, w \models B$. By the induction hypothesis, for all $w \in W \backslash\{v\}, \mathcal{M}^{\prime}, w \models B$. In particular since $u \in W \backslash\{v\}$ and $\mathcal{M}^{\prime}, u \models B$ iff $\mathcal{M}^{\prime}, u^{\prime \prime} \models B$, it follows that for all $w \in\left(W \cup\left\{u^{\prime \prime}\right\}\right) \backslash\{v\}$, $\mathcal{M}^{\prime}, w \models B$. Hence $\mathcal{M}^{\prime}, v \models[\neq] B$.

The proof of Proposition 5 does not use our present assumption about the finiteness of $\phi_{0}$. That is why, Proposition 5 also holds when $\phi_{0}$ is infinite.

Now take any model $\mathcal{M}=(W, V)$. The set $\left\{|u|_{V}: u \in W\right\}$ is finite since $\phi_{0}$ is finite and $\operatorname{card}\left(\left\{|u|_{V}: u \in W\right\}\right) \leq 2^{\operatorname{card}\left(\phi_{0}\right)}$. For each class $\mathcal{C}$ we define the set $\mathcal{C}^{1,2} \subseteq \mathcal{C}$ such that, if $\operatorname{card}(\mathcal{C})=1$ then $\mathcal{C}=\mathcal{C}^{1,2}$ otherwise $\operatorname{card}\left(\mathcal{C}^{1,2}\right)=2$. The elements of $\mathcal{C}^{1,2}$ can be seen as the representative elements of $\mathcal{C}$ (to be compared with the construction in the proof of Proposition 2). Define the model $\mathcal{M}^{\prime}=\mathcal{R}_{\mathcal{M}}=\left(W^{\prime}, V^{\prime}\right)$ such that,

- $W^{\prime}=\bigcup\left\{|u|_{V}^{1,2}: u \in W\right\}$ and,

- for all $P \in \phi_{0}, V^{\prime}(P)=V(P) \cap W^{\prime}$.

As a corollary of the previous proposition, for all $A \in \operatorname{FoR}\left(\phi_{0}\right), w \in W^{\prime}, \mathcal{M}, w \models$ $A$ iff $\mathcal{M}^{\prime}, w \models A$. Observe that $\operatorname{card}\left(W^{\prime}\right) \leq 2^{\operatorname{card}\left(\phi_{0}\right)} \times 2$. It is easy to show that

$$
\left\{\mathcal{R}_{\mathcal{M}}: \mathcal{M} \mathcal{E} \text {-model }\right\}=\left\{\mathcal{M}=(W, V) \mathcal{E} \text {-model }: \forall u \in W, \operatorname{card}\left(|u|_{V}\right) \leq 2\right\}
$$

This set of models contains exactly $3^{2^{\operatorname{card}\left(\phi_{0}\right)}}-1$ elements modulo isomorphic copies (the set of worlds of any model is non-empty). As a consequence,

Proposition 6. If $\phi_{0}$ is finite, deciding if a formula is satisfiable can be done in linear-time.

For each formula $A$, deciding if $A$ is satisfiable can be done in time at most $3^{2^{\operatorname{card}\left(\phi_{0}\right)}} \times K \times 2^{\operatorname{card}\left(\phi_{0}\right)+1} \times|A|$. As a consequence for any natural number $n \geq 1$, there is no $A \in \operatorname{FOR}\left(\phi_{0}\right)$ such that $A$ contains at most $n$ propositional variables and for all model $\mathcal{M}=(W, V), \mathcal{M} \models A$ iff $\operatorname{card}(W) \leq 2^{n+1}$. 


\section{Expressivity of $\mathcal{E}$ with Respect to S5}

The well-known modal logic S5 (see an introduction in [HC68]) shares with $\mathcal{E}$ the same set of models but the modal operators in S5 are denoted by $\square$ and $\diamond$. In the sequel, we write $\operatorname{FOR}^{\square}\left(\phi_{0}\right)$ to denote the set of formulas for S5. The satisfiability relation $\models$ is modified as follows:

$\mathcal{M}, w \models \square A$ iff for all $w^{\prime} \in W, \mathcal{M}, w^{\prime} \models A(\square$ behaves as $\mathcal{U})$.

Up to now, it appears that $\mathcal{E}$ and S5 have numerous common features: they share the same set of models and their respective satisfiability problem belongs to the same complexity class (even when the number of propositional variable is bounded). It is natural to wonder whether $\mathcal{E}$ and $\mathrm{S} 5$ have the same expressivity. First we show that $\mathcal{E}$ is at least as expressive as S5, i.e. for all $A \in \mathrm{FOR}^{\square}\left(\phi_{0}\right)$, there is $B \in \operatorname{FOR}\left(\phi_{0}\right)$ such that for all models $\mathcal{M}=(W, V), w \in W, \mathcal{M}, w \models_{S 5} A$ iff $\mathcal{M}, w \models_{\mathcal{E}} B$. Indeed consider the mapping $\mathcal{T}: \operatorname{FOR}^{\square}\left(\phi_{0}\right) \rightarrow \operatorname{FOR}\left(\phi_{0}\right)$ such that:

- for all $P \in \phi_{0}, \mathcal{T}(P)=P$,

- for all $A_{1}, A_{2} \in \mathrm{FOR}^{\square}\left(\phi_{0}\right), \mathcal{T}\left(A_{1} \wedge A_{2}\right)=\mathcal{T}\left(A_{1}\right) \wedge \mathcal{T}\left(A_{2}\right)$,

- for all $A \in \mathrm{FOR}^{\square}\left(\phi_{0}\right), \mathcal{T}(\neg A)=\neg \mathcal{T}(A)$ and $\mathcal{T}(\square A)=\mathcal{T}(A) \wedge[\neq] \mathcal{T}(A)$.

It is easy to show by induction on the structure of formulae that for all $A \in$ $\operatorname{FOR}^{\square}\left(\phi_{0}\right)$, for all models $\mathcal{M}=(W, V), w \in W, \mathcal{M}, w \models_{S 5} A$ iff $\mathcal{M}, w \models_{\mathcal{E}} \mathcal{T}(A)$. However the logic S5 is not so expressive as $\mathcal{E}$, i.e. there is $A \in \operatorname{FOR}\left(\phi_{0}\right)$ such that for all $B \in \mathrm{FOR}^{\square}\left(\phi_{0}\right)$ there is a model $\mathcal{M}=(W, V)$ and $w \in W$ such that $\mathcal{M}, w \models_{\mathcal{E}} A$ and $\mathcal{M}, w \models_{S 5} B$ have different values. Actually we show that it is not possible to express in S5 that a proposition holds in at least two worlds although this is possible in $\mathcal{E}$. We need to mention some simple properties about S5.

Proposition 7. Let $\mathcal{M}=(W, V)$ be a model, $u \in W$ and $A \in \mathrm{FOR}^{\square}\left(\phi_{0}\right)$. Take any $u^{\prime} \notin W$ and define a model $\mathcal{M}^{\prime}=\left(W \cup\left\{u^{\prime}\right\}, V^{\prime}\right)$ such that for all $P \in \phi_{0}$ occurring in $A, V^{\prime}(P)=V(P)$ if $u \notin V(P)$ otherwise $V^{\prime}(P)=V(P) \cup\left\{u^{\prime}\right\}$. Then for all $v \in W, \mathcal{M}, v \models_{S 5} A$ iff $\mathcal{M}^{\prime}, v \models_{S 5} A$.

The proof is omitted here but it has similarities with the proof of Proposition 5. So take any formula $A \in \mathrm{FOR}^{\square}\left(\phi_{0}\right)$, any model $\mathcal{M}=(W, V)$ and $u, u^{\prime} \in W$ such that for all $P \in \phi_{0}$ occurring in $A, u \in V(P)$ iff $u^{\prime} \in V(P)$. Define the model $\mathcal{M}^{\prime}=\left(W \backslash\left\{u^{\prime}\right\}, V^{\prime}\right)$ such that for all $P \in \phi_{0}, V^{\prime}(P)=V(P) \backslash\left\{u^{\prime}\right\}$. As a corollary of the previous proposition, for all $w \in W \backslash\left\{u^{\prime}\right\}, \mathcal{M}, w \models_{S 5} A$ iff $\mathcal{M}^{\prime}, w \models{ }_{S 5} A$.

Now consider the formula $A_{0}=P \wedge\langle\neq\rangle P$ for some $P \in \phi_{0}$ and the model $\left(W_{0}, V_{0}\right)$ such that $W_{0}=\{1,2\}$ and for all $Q \in \phi_{0}, V_{0}(Q)=W_{0}$. Obviously, $\mathcal{M}_{0}, 1 \models_{\mathcal{E}} A_{0}$. Suppose there is $B_{0} \in \mathrm{FOR}^{\square}\left(\phi_{0}\right)$ such that for all models $\mathcal{M}=$ $(W, V), w \in W, \mathcal{M}, w \models_{\mathcal{E}} A_{0}$ iff $\mathcal{M}, w \models_{S 5} B_{0}$. So $\mathcal{M}_{0}, 1 \models_{S 5} B_{0}$ and from the previous developments about $\mathrm{S} 5$, we have $\mathcal{M}_{0}^{\prime}, 1 \models_{S 5} B_{0}$ where $W_{0}^{\prime}=\{1\}$ and for all $Q \in \phi_{0}, V_{0}^{\prime}(Q)=W_{0}^{\prime}$ which leads to a contradiction since $\mathcal{M}_{0}^{\prime}, 1 \not \nvdash_{\mathcal{E}} A_{0}$. 
The comparison of the expressivity of $\mathcal{E}$ and S5 gives us the opportunity to notice that the problem of defining a sound and complete proof system for $\mathcal{E}$ is at least as difficult as the similar problem for S5 that has been intensively studied in the past (see e.g. [CW69,Fit77,DJP77,Sat80,FdC83,WW85,Gov95,MMO95]). Next section presents a prefixed tableau system for $\mathcal{E}$.

\section{A Complete Prefixed Tableau System for $\mathcal{E}$}

We shall define a prefixed tableaux following the methodology described in [Fit83,Fit77]. We use the types of modal formulae $(\nu, \pi, \alpha, \beta)$ defined in [Smu68,Fit83] (the symbol $[\neq]$-resp. $\langle\neq\rangle$ - replaces the symbol $\square$-resp. $\diamond$-). A prefixed formula is a pair $\langle k, A\rangle$ where $k \in \omega$ (set of natural numbers) called prefix and $A \in \operatorname{FOR}\left(\phi_{0}\right)$. We refer to a prefixed formula as atomic if it is of the form $\langle k, P\rangle$ or $\langle k, \neg P\rangle$ where $P \in \phi_{0}$. Figure 1 presents the different tableaux rules for $\mathcal{E}$. Observe that our system has similarities with the prefixed tableau system defined for S5 in [Fit83] (see also [Fey65]).

$(\pi) \frac{\langle k, \pi\rangle}{\left\langle k_{1}, \pi_{0}\right\rangle|\ldots|\left\langle k_{n}, \pi_{0}\right\rangle \mid\left\langle k^{\prime}, \pi_{0}\right\rangle}$ where $k_{1}, \ldots, k_{n}$ are the prefixes occurring on the branch, except $k$, and $k^{\prime}$ is a new prefix on the branch

$(\nu) \frac{\langle k, \nu\rangle}{\left\langle k^{\prime}, \nu_{0}\right\rangle}$ where $k^{\prime}$ is a prefix occurring on the branch different of $k$

$(\beta) \frac{\langle k, \beta\rangle}{\left\langle k, \beta_{1}\right\rangle \mid\left\langle k, \beta_{2}\right\rangle} \quad(\alpha) \frac{\langle k, \alpha\rangle}{\left\langle k, \alpha_{1}\right\rangle}$
$\left\langle k, \alpha_{2}\right\rangle$

Fig. 1. Tableaux rules

A branch is closed if there are contradictory prefixed formulae on that branch (for any $A \in \operatorname{FOR}\left(\phi_{0}\right), k \in \omega,\langle k, A\rangle$ and $\langle k, \neg A\rangle$ are contradictory). A tableau is closed if every branch is closed. A formula $A$ is said to have a closed tableau iff there is a closed tableau whose root is $\langle 1, \neg A\rangle$. Termination occurs when no operation is possible. A branch is open if it is terminated and not closed and a tableau is open if at least one branch is such. In the rest of this section we prove the soundness and the completeness of our tableau system with respect to the semantics of $\mathcal{E}$ by using the ideas of the proof technique developed in [Fit83] (chapter 8).

It is worth mentioning that if the $\pi$-rule presented in Figure 1 is replaced by $\frac{\langle k, \pi\rangle}{\left\langle k^{\prime}, \pi_{0}\right\rangle} k^{\prime}$ is a new prefix on the branch

then the system is not anymore sound with respect to the semantics of $\mathcal{E}$. For instance, in the system with this rule, the formula $\neg A$ has a closed tableau with

$$
A=((P \wedge[\neq] \neg P) \vee\langle\neq\rangle(P \wedge[\neq] \neg P)) \wedge\langle\neq\rangle P
$$

although $A$ is satisfiable. 


\subsection{Correctness}

Let $S$ be a set of prefixed formulae and let $\mathcal{M}=(W, V)$ be a model. By an interpretation of $S$ in the model $\mathcal{M}$ we mean an injective mapping

$$
\mathcal{I}:\{k:\langle k, B\rangle \in S\} \rightarrow W
$$

We say that $S$ is satisfiable under the interpretation $\mathcal{I}$ if for each $\langle k, A\rangle \in S$, $\mathcal{M}, \mathcal{I}(k) \models A$. We say $S$ is satisfiable if $S$ is satisfiable under some interpretation. We say that a branch of a tableau is satisfiable if the set of prefixed formulae on it, is satisfiable. A tableau is satisfiable if some branch is.

Lemma 1. Suppose $\mathrm{T}$ is a prefixed tableau that is satisfiable. Let $\mathrm{T}^{\prime}$ be the tableau that results from a single tableau rule being applied to $\mathrm{T}$. Then $\mathrm{T}^{\prime}$ is also satisfiable.

Proof. If $\mathrm{T}$ is satisfiable because the branch $\mathrm{B}$ is satisfiable and if our tableau rule is not applied on branch $\mathrm{B}$ then $\mathrm{B}$ is still present in $\mathrm{T}^{\prime}$ and still satisfiable. So consider the case where the tableau rule in question is applied to a satisfiable branch $\mathrm{B}$ of $\mathrm{T}$. If the tableau rule is either $\alpha$ or $\beta$ it is easy to produce a satisfiable branch of $\mathrm{T}^{\prime}$. Suppose that $\mathrm{B}$ is satisfiable under the interpretation $\mathcal{I}$ in the model $\mathcal{M}=(W, V)$ and one of the rule $(\pi),(\nu)$ is applied.

First the $\nu$-rule. Suppose $\langle k, \nu\rangle$ occurs on $\mathrm{B}$, and $\left\langle k^{\prime}, \nu_{0}\right\rangle$ is added to the end of $\mathrm{B}\left(k^{\prime} \neq k\right)$. Since $k^{\prime}$ was used on $\mathrm{B}, \mathcal{I}$ is already defined for $k^{\prime}$ and $\mathcal{I}(k) \neq \mathcal{I}\left(k^{\prime}\right)(\mathcal{I}$ is injective). Since B is satisfiable under $\mathcal{I}$ and $\langle k, \nu\rangle$ occurs in $\mathrm{B}, \mathcal{M}, \mathcal{I}(k) \models \nu$. It follows that $\mathcal{M}, \mathcal{I}\left(k^{\prime}\right) \models \nu_{0}$. Thus the new branch is still satisfiable under the same interpretation $\mathcal{I}$.

Second the $\pi$-rule. Suppose $\langle k, \pi\rangle$ occurs on $\mathrm{B}$, and for all $i \in\{1, \ldots, n\}$, $\mathrm{B}_{i}=\mathrm{B} \cup\left\{\left\langle k_{i}, \pi_{0}\right\rangle\right\}$ and $\mathrm{B}_{n+1}=\mathrm{B} \cup\left\{\left\langle k^{\prime}, \pi_{0}\right\rangle\right\}$ where $k_{1}, \ldots, k_{n}$ are the prefixes occurring on $\mathrm{B}$ (different of $k$ ) and $k^{\prime}$ is a new prefix. By hypothesis, $\mathcal{M}, \mathcal{I}(k) \models \pi$. There is $w^{\prime} \in W \backslash\{\mathcal{I}(k)\}$ such that $\mathcal{M}, w^{\prime} \models \pi_{0}$. If there is $i \in\{1, \ldots, n\}$ such that $\mathcal{I}\left(k_{i}\right)=w^{\prime}$ then $\mathrm{B}_{i}$ is satisfiable. Otherwise, consider $\mathcal{I}^{\prime}:\left\{k, k_{1}, \ldots, k_{n}, k^{\prime}\right\} \rightarrow W$ such that for all $k^{\prime \prime} \in\left\{k, k_{1}, \ldots, k_{n}\right\}, \mathcal{I}^{\prime}\left(k^{\prime \prime}\right)=\mathcal{I}\left(k^{\prime \prime}\right)$ and $\mathcal{I}^{\prime}\left(k^{\prime}\right)=w^{\prime}$. It is easy to check that $\mathcal{I}^{\prime}$ is injective and that $\mathrm{B}_{n+1}$ is satisfiable under $\mathcal{I}^{\prime}$.

Proposition 8. If $A \in \operatorname{FOR}\left(\phi_{0}\right)$ has a closed prefixed tableau then $A$ is valid.

Proof. Similar to the proof of Theorem 3.2 in [Fit83] (p.400).

\subsection{Completeness}

Let $A$ be a formula. As done in [Fit83], we define a systematic attempt to produce a proof of $A$. The procedure is in stages and the stage 1 consists in placing $\langle 1, \neg A\rangle$ at the root. Now suppose $n$ stages of the construction have been done. If the tableau is closed then we stop. Similarly if every occurrence of a prefixed formula is finished then we stop. Otherwise we go on. Any stage $n+1$ consists in choosing an occurrence of a prefixed formula $\langle k, B\rangle$ as high up in the tree as possible (as 
close to the origin as possible) that has not been finished. If $\langle k, B\rangle$ is atomic then the occurrence is declared finished. This ends the stage $n+1$ otherwise we extend the tableau as follows. For each open branch B through the occurrence of $\langle k, B\rangle$ :

- If $\langle k, B\rangle$ is of the form $\langle k, \alpha\rangle$ add $\left\langle k, \alpha_{1}\right\rangle$ and $\left\langle k, \alpha_{2}\right\rangle$ to the end of B.

- If $\langle k, B\rangle$ is of the form $\langle k, \beta\rangle$ split the end of branch $\mathrm{B}$ and add $\left\langle k, \beta_{1}\right\rangle$ to the end of one sub-branch and $\left\langle k, \beta_{2}\right\rangle$ to the end of the other one.

- If $\langle k, B\rangle$ is of the form $\langle k, \nu\rangle$ then for each prefix $k^{\prime} \neq k$ used on $\mathrm{B}$ add $\left\langle k^{\prime}, \nu_{0}\right\rangle$ to the end of $\mathrm{B}$, after which add a fresh occurrence of $\langle k, \nu\rangle$ to the end of $\mathrm{B}$.

- If $\langle k, B\rangle$ is of the form $\langle k, \pi\rangle$ then split the end of $\mathrm{B}$ in $m+1$ sub-branches $\mathrm{B}_{1}, \ldots, \mathrm{B}_{m+1}$ where $k_{1}, \ldots, k_{m}$ are the prefixes different of $k$ occurring in $\mathrm{B}$ and $k^{\prime}$ is a new prefix not occurring in $\mathrm{B}$ and for all $i \in\{1, \ldots, m\}$ add at the end of each sub-branch $\mathrm{B}_{i}\left\langle k_{i}, \pi_{0}\right\rangle$ and at the end of $\mathrm{B}_{m+1},\left\langle k^{\prime}, \pi_{0}\right\rangle$.

Having done this for each branch $B$ through the particular occurrence of $\langle k, B\rangle$ being considered declare that occurrence of $\langle k, B\rangle$ finished. This ends stage $n+1$.

Definition 2. Let $S$ be a set of prefixed formulae. We say $S$ is downwardsaturated if:

- for all $P \in \phi_{0}, k \in \omega,\{\langle k, P\rangle,\langle k, \neg P\rangle\} \nsubseteq S$

- if $\langle k, \alpha\rangle \in S$ then $\left\{\left\langle k, \alpha_{1}\right\rangle,\left\langle k, \alpha_{2}\right\rangle\right\} \subseteq S$

- if $\langle k, \beta\rangle \in S$ then either $\left\langle k, \beta_{1}\right\rangle \in S$ or $\left\langle k, \beta_{2}\right\rangle \in S$

- if $\langle k, \nu\rangle \in S$ then for all $k^{\prime} \neq k$ occurring in $S$ we have $\left\langle k^{\prime}, \nu_{0}\right\rangle \in S$

- if $\langle k, \pi\rangle \in S$ then there is $k^{\prime} \neq k$ occurring in $S$ such that $\left\langle k^{\prime}, \pi_{0}\right\rangle \in S$

Lemma 2. If $S$ is downward-saturated then $S$ is satisfiable in a model whose worlds are the prefixes occurring in $S$.

Proof. Assume $S$ is downward-saturated. Let $\mathcal{M}=(W, V)$ be the model such that,

$$
\begin{aligned}
& -W=\{k:\langle k, B\rangle \in S\} \\
& \text { - for all } P \in \phi_{0} V(P)=\{k:\langle k, P\rangle \in S\} .
\end{aligned}
$$

It can be shown by induction on the structure of the formulae that for every formula $B$ and every prefix $k$, if $\langle k, B\rangle \in S$ then $\mathcal{M}, k \models B$ (and therefore $S$ is satisfiable under the identity function in $\mathcal{M})$. Assume $\langle k, P\rangle \in S$ with $P \in \phi_{0}$. By definition of $V,\langle k, P\rangle \in S$ iff $k \in V(P)$ iff $\mathcal{M}, k \models P$ and therefore $\mathcal{M}, k \models P$. The cases when $B$ has the form $\alpha$ or $\beta$ are omitted here. Assume $\langle k, \nu\rangle \in S$. Since $S$ is downward-saturated for all $k^{\prime} \neq k$ occurring in $S,\left\langle k^{\prime}, \nu_{0}\right\rangle \in S$. By the induction hypothesis, for all $k^{\prime} \in W \backslash\{k\}, \mathcal{M}, k^{\prime} \models \nu_{0}$. Hence $\mathcal{M}, k \models \nu$. Assume $\langle k, \pi\rangle \in S$. Since $S$ is downward-saturated there is $k^{\prime} \neq k$ occurring in $S$ such that $\left\langle k^{\prime}, \pi_{0}\right\rangle \in S$. By the induction hypothesis, there is $k^{\prime} \in W \backslash\{k\}$ such that $\mathcal{M}, k^{\prime} \models \pi_{0}$. Hence $\mathcal{M}, k \models \pi$. 
Proposition 9. If $A \in \operatorname{FOR}\left(\phi_{0}\right)$ is valid then $A$ has a closed prefixed tableau built with the rules presented in Figure 1.

Proof. Suppose $A$ has no closed prefixed tableau. So the systematic procedure does not generate a closed tableau. We build a tableau with this procedure by considering $\langle 1, \neg A\rangle$ at the root. If the procedure terminates then the tableau contains a non-closed branch. If the procedure does not terminate, by König's Lemma (every infinite, finitely generated tree has an infinite branch), there is an infinite non-closed branch. The systematic procedure guarantees that the nonclosed branch B is downward-saturated. By Lemma 2, B is satisfiable. Since $\langle 1, \neg A\rangle \in \mathrm{B}$, there is a model $\mathcal{M}=(W, V)$ and $w \in W$ such that $\mathcal{M}, w \models \neg A$, which leads to a contradiction.

$A$ decision procedure The systematic proof procedure is modified in such a way that, if $\langle k, B\rangle$ has to be added on the branch $\mathrm{B}$ and if $\langle k, B\rangle$ is already on $B$ then no new occurrence is added. When the procedure ends either we get a closed prefixed tableau, or we get a counter-model (see the previous propositions). Actually the procedure terminates. Suppose that the procedure does not terminate by trying to prove the validity of $A$. By König's Lemma, an infinite branch $\mathrm{B}_{0}$ is built. If $\langle k, B\rangle \in \mathrm{B}_{0}$ then $B \in \operatorname{sub}\left(\operatorname{sub}(\neg A) \cup\left\{\neg A_{1}, \neg A_{2}\right.\right.$ : $\left.\left.\left\{\neg\left(A_{1} \wedge A_{2}\right), \neg[\neq] A_{1}, \neg\langle\neq\rangle A_{1},\right\} \cap \operatorname{sub}(\neg A) \neq \emptyset\right\}\right)=S$. Since $S$ is finite then each prefix appears a finite number of times. So $\mathrm{B}_{0}$ contains an infinite number of prefixes. Let $n$ be the greatest natural number in $\left\{|B|: \exists\langle k, B\rangle \in \mathrm{B}_{0}\right\}$ such that $\left\{k^{\prime}:\left\langle k^{\prime}, B\right\rangle \in \mathrm{B}_{0},|B| \geq n\right\}$ is infinite. We have $n \neq|\neg A|$ since the only prefixed formula of the form $\langle k, B\rangle$ with $|B|=|\neg A|$ occurring in $\mathrm{B}_{0}$ is $\langle 1, \neg A\rangle$. Since the application of the rules in Figure 1 strictly decreases the length of the formulae this leads to a contradiction with the minimality of $n$.

\section{$6 \quad$ Extensions}

\subsection{Logical Consequence}

The rule for introducing a set of global assumptions $S$ into the tableau proofs is straightforward:

$\overline{\left\langle k_{i}, A\right\rangle}$ for some $A \in S, k_{i}$ occurring on the branch

Proposition 10. Let $S \subseteq \operatorname{FOR}\left(\phi_{0}\right), A \in \operatorname{FOR}\left(\phi_{0}\right)$. There is a closed prefixed tableau whose root is $\langle 1, \neg A\rangle$ using the members of $S$ as global assumptions iff $S \models A$.

Proof. By an easy modification of the proof of Theorem 8.1 in [Fit83].

Observe that for all finite sets $S=\left\{A_{1}, \ldots, A_{n}\right\} \subseteq \operatorname{FOR}\left(\phi_{0}\right), S \models A$ iff

$$
A_{1} \wedge \ldots \wedge A_{n} \wedge[\neq]\left(A_{1} \wedge \ldots \wedge A_{n}\right) \Rightarrow A \wedge[\neq] A
$$

is valid (this can related to Corollary 5.3 in [GP92]). So the problem of determining whether $S \models A$ holds where $S$ is finite set of formulae and $A \in \operatorname{FOR}\left(\phi_{0}\right)$ is co-NP-complete (remember the validity problem for $\mathcal{E}$ is co-NP-complete). The logic S5 also shares this property (see e.g. [FHV92]). 


\subsection{An Extended Calculus}

We extend the language $\mathrm{L}$ into the language $\mathrm{L}^{*}$ admitting the modal operators $\mathcal{U}$ and $\langle!\rangle$. We write $\operatorname{FOR}^{*}\left(\phi_{0}\right)$ to denote the smallest extension of $\operatorname{FOR}\left(\phi_{0}\right)$ closed under $\mathcal{U}$ and $\langle!\rangle$. The relation $\models$ is naturally extended as follows:

- $\mathcal{M}, w \models \mathcal{U} A$ iff for all $w^{\prime} \in W, \mathcal{M}, w^{\prime} \models A$

- $\mathcal{M}, w \models\langle!\rangle A$ iff there is a unique $w^{\prime} \in W$ such that $\mathcal{M}, w^{\prime} \models A$

The addition of the operators $\langle!\rangle$ and $\mathcal{U}$ with the above semantics does not increase the expressivity of $\mathcal{E}$. However these operators are quite natural to express properties. Consider the tableau system composed of the rules in Figure 1 and 2 .

$(\mathcal{U}) \frac{\langle k, \mathcal{U} A\rangle}{\left\langle k_{i}, A\right\rangle} \quad(\neg \mathcal{U}) \frac{\langle k, \neg \mathcal{U} A\rangle}{\left\langle k_{1}, \neg A\right\rangle|\ldots|\left\langle k_{n}, \neg A\right\rangle \mid\left\langle k^{\prime}, \neg A\right\rangle}$

$(\langle!\rangle) \frac{\langle k,\langle!\rangle A\rangle}{\left\langle k_{1}, A \wedge[\neq] \neg A\right\rangle|\ldots|\left\langle k_{n}, A \wedge[\neq] \neg A\right\rangle \mid\left\langle k^{\prime}, A \wedge[\neq] \neg A\right\rangle}$

$(\neg\langle!\rangle) \frac{\langle k, \neg\langle!\rangle A\rangle}{\langle k, \mathcal{U} \neg A\rangle\left|\left\langle k_{1}, A \wedge\langle\neq\rangle A\right\rangle\right| \ldots\left|\left\langle k_{n}, A \wedge\langle\neq\rangle A\right\rangle\right|\left\langle k^{\prime}, A \wedge\langle\neq\rangle A\right\rangle}$

$k^{\prime}$ is a new prefix on the branch, $\left\{k_{1}, \ldots, k_{n}\right\}$ is the set of prefixes occurring on the branch.

Fig. 2. Additional tableaux rules

Proposition 11. If $A \in \mathrm{FOR}^{*}\left(\phi_{0}\right)$ has a closed prefixed tableau built with the rules in Figure 1 and 2 then $A$ is valid.

Proof. The proof follows the lines of the proof of Lemma 1 and Proposition 8. By way of example we show that if a branch B is satisfiable under the interpretation $\mathcal{I}$ in the model $\mathcal{M}=(W, V)$ and if the $\neg\langle!\rangle$-rule is applied on $\mathrm{B}$ then one of the new branches is satisfiable. Suppose $\langle k, \neg\langle!\rangle B\rangle$ occurs on $\mathrm{B}, \mathrm{B}_{n+1}=$ $\mathrm{B} \cup\{\langle k, \mathcal{U} \neg B\rangle\}, \mathrm{B}_{n+2}=\mathrm{B} \cup\left\{\left\langle k^{\prime}, B \wedge\langle\neq\rangle B\right\rangle\right\}$ and for all $i \in\{1, \ldots, n\}, \mathrm{B}_{i}=$ $\mathrm{B} \cup\left\{\left\langle k_{i}, B \wedge\langle\neq\rangle B\right\rangle\right\}$ where $k_{1}, \ldots, k_{n}$ are the prefixes occurring on $\mathrm{B}$ (including $k)$ and $k^{\prime}$ is a new prefix. By hypothesis, $\mathcal{M}, \mathcal{I}(k) \models \neg\langle!\rangle B$. We have either $\operatorname{card}(\{u \in W: \mathcal{M}, u \models B\})=0$ or $\operatorname{card}(\{u \in W: \mathcal{M}, u \models B\})>1$. When the first possibility holds, $\mathcal{M}, \mathcal{I}(k) \models \mathcal{U} \neg B$. Hence $\mathrm{B}_{n+1}$ is satisfiable under $\mathcal{I}$ in $\mathcal{M}$. Otherwise, there are $u_{1}, u_{2} \in W\left(u_{1} \neq u_{2}\right)$ such that $\mathcal{M}, u_{1} \models B$ and $\mathcal{M}, u_{2} \models B$. If for some $i \in\{1, \ldots, n\}, \mathcal{I}\left(k_{i}\right) \in\left\{u_{1}, u_{2}\right\}$ then $\mathcal{M}, \mathcal{I}\left(k_{i}\right) \models B \wedge\langle\neq\rangle B$. Hence $\mathrm{B}_{i}$ is satisfiable under $\mathcal{I}$ in $\mathcal{M}$. Otherwise, consider $\mathcal{I}^{\prime}:\left\{k_{1}, \ldots, k_{n}, k^{\prime}\right\} \rightarrow W$ such that for all $i \in\{1, \ldots, n\}, \mathcal{I}^{\prime}\left(k_{i}\right)=\mathcal{I}\left(k_{i}\right)$ and $\mathcal{I}^{\prime}\left(k^{\prime}\right)=u_{1}$. So $\mathrm{B}_{m+2}$ is satisfiable under $\mathcal{I}^{\prime}$ in $\mathcal{M}$ since $\mathcal{M}, \mathcal{I}^{\prime}\left(k^{\prime}\right) \models B \wedge\langle\neq\rangle B$. It is easy to check that $\mathcal{I}^{\prime}$ is injective.

The systematic procedure is extended as follows: 
- If $\langle k, B\rangle$ is of the form $\left\langle k, \mathcal{U} A_{1}\right\rangle$ then for each prefix $k_{i}$ used on $\mathrm{B}$ add $\left\langle k_{i}, A_{1}\right\rangle$ to the end of $\mathrm{B}$, after which add a fresh occurrence of $\left\langle k, \mathcal{U} A_{1}\right\rangle$ to the end of $\mathrm{B}$.

- If $\langle k, B\rangle$ is of the form $\left\langle k, \neg \mathcal{U} A_{1}\right\rangle$ (resp. $\left\langle k,\langle!\rangle A_{1}\right\rangle$ ) then split the end of $\mathrm{B}$ in $m+1$ sub-branches $\mathrm{B}_{1}, \ldots, \mathrm{B}_{m+1}$ where $k_{1}, \ldots, k_{m}$ are the prefixes occurring in $\mathrm{B}$ and $k^{\prime}$ is a new prefix not occurring in $\mathrm{B}$ and for all $i \in\{1, \ldots, m\}$ add at the end of the sub-branch $\mathrm{B}_{i}\left\langle k_{i}, \neg A_{1}\right\rangle$ (resp. $\left\langle k_{i}, A_{1} \wedge[\neq] \neg A_{1}\right\rangle$ ) and at the end of $\mathrm{B}_{m+1},\left\langle k^{\prime}, \neg A_{1}\right\rangle$ (resp. $\left\langle k^{\prime}, A_{1} \wedge[\neq] \neg A_{1}\right\rangle$ ).

- If $\langle k, B\rangle$ is of the form $\left\langle k, \neg\langle!\rangle A_{1}\right\rangle$ then split the end of $\mathrm{B}$ in $m+2$ subbranches $\mathrm{B}_{1}, \ldots, \mathrm{B}_{m+1}$ where $k_{1}, \ldots, k_{m}$ are the prefixes occurring in $\mathrm{B}$ and $k^{\prime}$ is a new prefix not occurring in $\mathrm{B}$ and for all $i \in\{1, \ldots, m\}$ add at the end of the sub-branch $\mathrm{B}_{i}\left\langle k_{i}, A_{1} \wedge\langle\neq\rangle A_{1}\right\rangle$, at the end of $\mathrm{B}_{m+1},\left\langle k^{\prime}, A_{1} \wedge\langle\neq\rangle A_{1}\right\rangle$ and at the end of $\mathrm{B}_{m+2},\left\langle k, \mathcal{U} \neg A_{1}\right\rangle$.

The definition of a downward-saturated set in Definition 2 is augmented with the conditions:

- if $\langle k, \mathcal{U} A\rangle \in S$ then for all $k^{\prime}$ occurring in $S$ we have $\left\langle k^{\prime}, A\right\rangle \in S$.

- if $\langle k, \neg \mathcal{U} A\rangle \in S$ then there is $k^{\prime}$ occurring in $S$ such that $\left\langle k^{\prime}, \neg A\right\rangle \in S$.

- if $\langle k,\langle!\rangle A\rangle \in S$ then $\operatorname{card}\left(\left\{k^{\prime}:\left\langle k^{\prime}, A\right\rangle \in S\right\}\right)=1$.

- if $\langle k, \neg\langle!\rangle A\rangle \in S$ then $\operatorname{card}\left(\left\{k^{\prime}:\left\langle k^{\prime}, A\right\rangle \in S\right\}\right) \neq 1$.

Lemma 3. If $S$ is downward-saturated (with the extensions) then $S$ is satisfiable in a model whose worlds are simply the prefixes occurring in members of $S$.

Proof. The proof follows the lines of the proof of Lemma 2. Assume $S$ is downwardsaturated and let $\mathcal{M}=(\{k:\langle k, B\rangle \in S\}, V)$ be the model such that for all $P \in \phi_{0}, V(P)=\{k:\langle k, P\rangle \in S\}$. By way of example, we show that if $\langle k, \mathcal{U} A\rangle \in S$ then $\mathcal{M}, k \models \mathcal{U} A$ (in the induction step). Since $S$ is downwardsaturated, for all $k^{\prime}$ occurring in $S,\left\langle k^{\prime}, A\right\rangle \in S$. By the induction hypothesis, for all $k^{\prime}$ occurring in $S, \mathcal{M}, k^{\prime} \models A$. Hence $\mathcal{M}, k \models \mathcal{U} A$.

Finally,

Proposition 12. If $A \in \operatorname{FOR}^{*}\left(\phi_{0}\right)$ is valid then $A$ has a closed prefixed tableau built with the rules presented in Figure 1 and 2.

Now we give a systematic proof procedure using the rules in Figure 1 and 2 that constitutes a decision procedure for the satisfiability problem with the extended language $\mathrm{L}^{*}$. First some definitions are needed. We define $s u b^{*}(A)$ as the smallest set $\Sigma$ such that

$-A \in \Sigma$,

- if $\left\{\neg A_{1},[\neq] A_{1},\langle\neq\rangle A_{1}, \mathcal{U} A\right\} \cap \Sigma \neq \emptyset$ then $\left\{A_{1}, \neg A_{1}\right\} \subseteq \Sigma$

- if $\left\{A_{1} \vee A_{2}, A_{1} \wedge A_{2}\right\} \cap \Sigma \neq \emptyset$ then $\left\{A_{1}, A_{2}, \neg A_{1}, \neg A_{2}\right\} \subseteq \Sigma$

- if $\langle!\rangle A_{1} \in \Sigma$ then $\left\{A_{1} \wedge[\neq] \neg A_{1}, A_{1} \wedge\langle\neq\rangle A_{1}, \mathcal{U} \neg A_{1}\right\} \subseteq \Sigma$ 
For all $A \in \operatorname{FOR}^{*}\left(\phi_{0}\right), s u b^{*}(A)$ is finite. For all $B \in s u b^{*}(A)$ we write $|B|^{*}$ (the $\langle!\rangle$-degree of $B$ ) to denote the pair $\left\langle|B|^{\langle!\rangle},|B|\right\rangle$ where $|B|^{\langle!\rangle}$is the maximal number of occurrences of $\langle!\rangle$ that dominate some propositional variable of $B$.

We also consider here the modified systematic proof procedure presented in Section 5.2. We shall show that the procedure terminates providing a decision procedure. Suppose that the procedure does not terminate by trying to prove $A$. By König's Lemma, an infinite branch $\mathrm{B}_{0}$ is built. If $\langle k, B\rangle \in \mathrm{B}_{0}$ then $B \in$ $s u b^{*}(\neg A)$. Since $s u b^{*}(\neg A)$ is finite then each prefix appears a finite number of times. So $\mathrm{B}_{0}$ contains an infinite number of prefixes. Let $\left\langle n_{1}, n_{2}\right\rangle$ be a maximal pair in $\left\{|B|^{*}: \exists\langle k, B\rangle \in \mathrm{B}_{0}\right\}$ with respect to $\leq$, the well-founded lexicographic order on $\omega^{2}$, such that $\left\{k^{\prime}:\left\langle k^{\prime}, B\right\rangle \in \mathrm{B}_{0},|B|^{*} \geq\left\langle n_{1}, n_{2}\right\rangle\right\}$ is infinite. It follows that for all $\left\langle m_{1}, m_{2}\right\rangle \in S$ where

$$
S=\left\{\left|B^{\prime}\right|^{*}: \exists\left\langle k^{\prime}, B^{\prime}\right\rangle \in \mathrm{B}_{0},\left|B^{\prime}\right|^{*}>\left\langle n_{1}, n_{2}\right\rangle\right\}
$$

the set $\left\{k^{\prime}:\left\langle k^{\prime}, B\right\rangle \in \mathrm{B}_{0},|B|^{*} \geq\left\langle m_{1}, m_{2}\right\rangle\right\}$ is finite (by the minimality of $\left.\left\langle n_{1}, n_{2}\right\rangle\right)$. Observe that $S$ is finite since for all $\left\langle k^{\prime}, B\right\rangle \in \mathrm{B}_{0}, B \in \operatorname{sub}^{*}(\neg A)$. Hence,

$$
\operatorname{card}(S) \leq \operatorname{card}\left(|B|^{*}: B \in \operatorname{sub}^{*}(\neg A)\right)
$$

Since the application of the rules in the Figure 1 and 2 strictly decreases the $\langle!\rangle$-degree of the prefixed formulae, for all $\langle k, B\rangle \in \mathrm{B}_{0}$ such that $|B|^{*} \geq\left\langle n_{1}, n_{2}\right\rangle$ there is $\left\langle k^{\prime}, B^{\prime}\right\rangle \in \mathrm{B}_{0}$ such that $\langle k, B\rangle$ is obtained from $\left\langle k^{\prime}, B^{\prime}\right\rangle$ by application of a rule such that $\left|B^{\prime}\right|^{*}>|B|^{*}$. Since only a finite set of prefixed formulae is obtained with a single inference rule and since each prefixed formula can be the premise of a unique tableau rule, the set $\left.\left\{k^{\prime}:\left\langle k^{\prime}, B\right\rangle \in \mathrm{B}_{0},|B|^{*}\right\rangle\left\langle n_{1}, n_{2}\right\rangle\right\}$ is also infinite which leads to a contradiction by the finiteness of $S$ and by the minimality of $\left\langle n_{1}, n_{2}\right\rangle$.

Example Let us prove the validity of $\langle!\rangle A \wedge\langle!\rangle B \wedge \mathcal{U}(A \Rightarrow B) \Rightarrow\langle!\rangle(A \wedge B)$ using the extended version of our tableau proof system. In Figure 3 the symbol \& (resp. [\#],,$- X)$ corresponds to the symbol $\wedge$ (resp. the symbol $[\neq]$, the symbol $\neg$, the fact that the branch is closed). The case analysis are represented either by $\left\langle k_{1}, A_{1}\right\rangle \mid\left\langle k_{2}, A_{2}\right\rangle$ or by $\overline{\left\langle k_{1}, A_{1}\right\rangle\left|\left\langle k_{2}, A_{2}\right\rangle\right|\left\langle k_{3}, A_{3}\right\rangle}$. Moreover each prefixed formula $\left\langle k, A^{\prime}\right\rangle$ is simply written $k, A^{\prime}$.

\section{Concluding Remarks}

In this paper we have defined a sound and complete prefixed tableau system for the logic of elsewhere $\mathcal{E}$ defined by von Wright and for which an Hilbert-style system has been proved to be sound and complete in [Seg81] (see Section 5). Our system uses a technique similar to the Feys's prefixed tableau system for S5 and it can be easily implemented in any existing tableau-based prover. It is part of our future work to implement this proof system in the ATINF tableau-based theorem prover for modal logics (see e.g. [Dem95]). We believe that it would be also of great interest to define prefixed tableau systems for logics involving the difference 


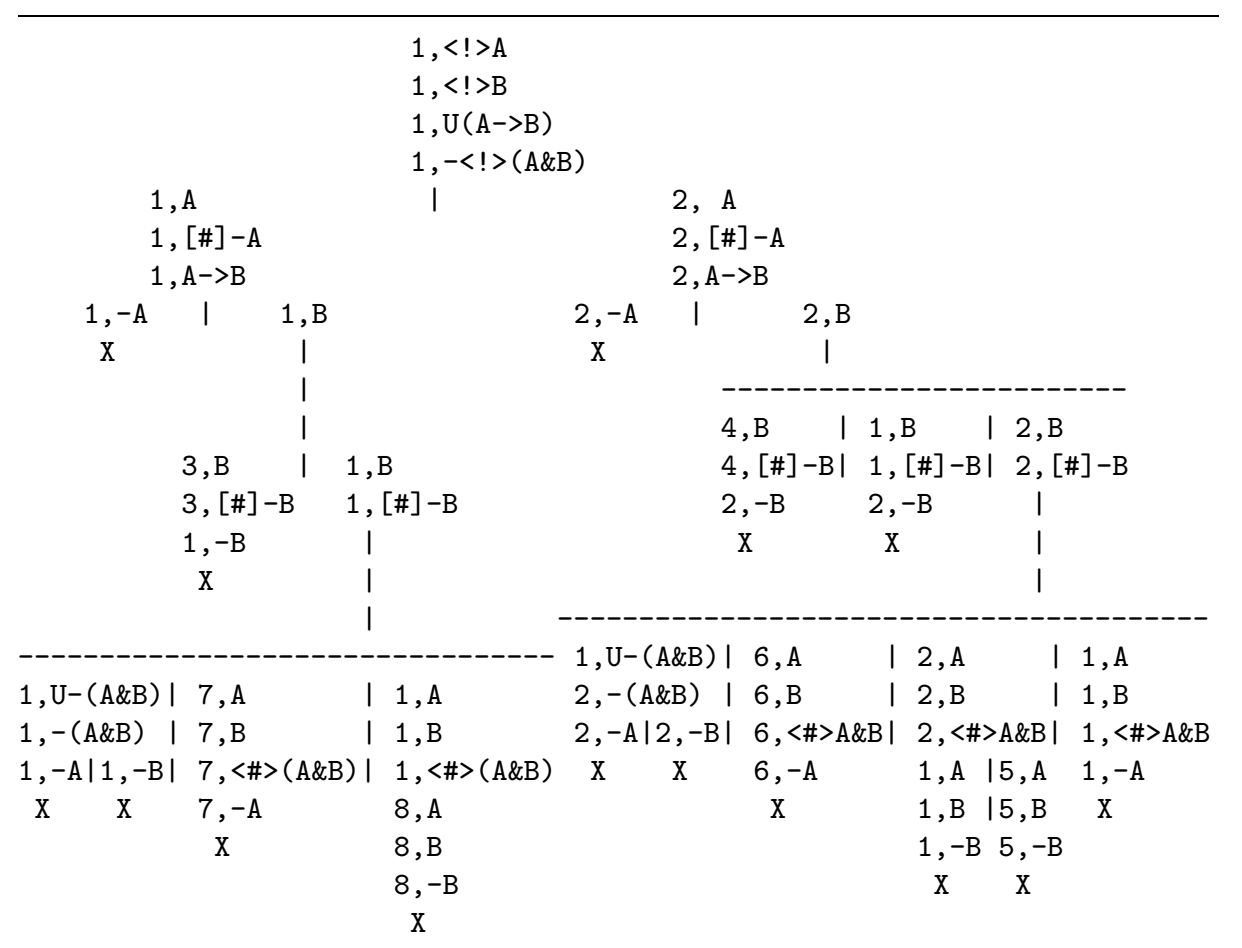

Fig. 3. An example of tableau proof 
operator and different other modal operators such as the different logics studied for example in [GPT87,GG93,GP92,Rij92]. For instance, it is possible to admit in the same language the classical modal operator $\square$ and the difference operator $[\neq]$. Apart from the tableau rules for the modal operator $\square$, a $\pi$-rule for the difference operator $\langle\neq\rangle$ of the form

$\frac{\langle\sigma,\langle\neq\rangle, A\rangle}{\left\langle\sigma_{1}, A\right\rangle|\ldots|\left\langle\sigma_{n}, A\right\rangle \mid\left\langle k^{\prime}, A\right\rangle}$ where $\sigma_{1}, \ldots, \sigma_{n}$ are the prefixes occurring on the branch (different of $\sigma$ ) and $k^{\prime}$ is a new prefix of length 1 on the branch

might not be sound since we have to guarantee that the interpretations of $\sigma$ and $k^{\prime}$ are different worlds (for example). So, the definition of complete prefixed tableau systems for the logics studied in the previously mentioned works remains an open problem.

Another aspect of our work has been to consider the computational complexity of the satisfiability problem for $\mathcal{E}$. We have shown that a (better) different bound than the one in [Rij92] can be given for the size of the model satisfying a given formula. Moreover, when the number of propositional variables is bounded we have shown that at most $3^{2^{\operatorname{card}\left(\phi_{0}\right)}}$ models have to considered and each model has at most $2^{\operatorname{card}\left(\phi_{0}\right)+1}$ worlds (see Section 3 ). Hence in this case the satisfiability problem for $\mathcal{E}$ is linear-time, as it is also true for propositional logic S5. Moreover we have shown that the logics S5 and $\mathcal{E}$ have different expressive powers although they share numerous common features (see Section 4).

In conclusion, this work remains a first step to define sound and complete prefixed tableau systems and to characterize the computational complexity of problems for modal logics with some enriched languages. Investigations in that direction appears to be a promising research area in order to combine the expressivity of these logics with the (possible) efficiency of their mechanization.

Acknowledgments The author thanks Ricardo Caferra and the anonymous referees for their precious remarks and suggestions about this work.

\section{References}

[Cat91] Laurent Catach. TABLEAux : a general theorem prover for modal logics. Journal of Automated Reasoning, 7:489-510, 1991.

[Coo71] S. Cook. The complexity of theorem-proving procedures. In Third Annual ACM Symposium on Theory of Computing, pages 151-158, 1971.

[CW69] J. Corcoran and G. Weaver. Logical consequence in modal logic: natural deduction in S5. Notre Dame Journal of Formal Logic, 10(4):370-384, October 1969.

[Dem95] Stéphane Demri. Uniform and non uniform strategies for tableaux calculi for modal logics. Journal of Applied Non Classical Logics, 5(1):77-96, January 1995.

[DJP77] B. Davidson, F. C. Jackson, and R. Pargetter. Modal trees for T and S5. Notre Dame Journal of Formal Logic, 18(4):602-606, October 1977.

[DSVEB90] B. De Smit and P. Van Emde Boas. The complexity of the modal difference operator in propositional logic, 1990. Unpublished note, University of Amsterdam. 
[FdC83] L. Fariñas del Cerro. Un principe de résolution en logique modale. RAIRO, 18:161-170, 1983.

[Fey65] R. Feys. Modal logics. B. Gauthier-Villars, Paris, 1965.

[FHV92] R. Fagin, J. Y. Halpern, and M. Y. Vardi. What is an inference rule? Journal of Symbolic Logic, 57(3):1018-1045, September 1992.

[Fit77] Melvin Fitting. A tableau system for propositional S5. Notre Dame Journal of Formal Logic, 18(2):292-294, 1977.

[Fit83] Melvin Fitting. Proof methods for modal and intuitionistic logics. D. Reidel Publishing Co., 1983.

[Fit88] Melvin Fitting. First-order modal tableaux. Journal of Automated Reasoning, 4:191-213, 1988.

[GG93] George Gargov and V. Goranko. Modal logic with names. Journal of Philosophical Logic, 22(6):607-636, December 1993.

[Gor90] Valentin Goranko. Modal definability in enriched languages. Notre Dame Journal of Formal Logic, pages 81-105, 1990.

[Gor92] R. P. Goré. Cut-free sequents and tableau systems for propositional normal modal logics. PhD thesis, University of Cambridge, England, May 1992.

[Gov95] G. Governatori. Labelled tableaux for multi-modal logics. In P. Baumgartner, Reiner Hähnle, and J. Posegga, editors, Workshop on Theorem Proving with Analytic Tableaux and Related Methods, pages 79-94. LNAI 918, Springer-Verlag, May 1995.

[GP92] Valentin Goranko and Solomon Passy. Using the universal modality: gains and questions. Journal of Logic Computation, 2(1):5-30, 1992.

[GPT87] George Gargov, Solomon Passy, and Tinko Tinchev. Modal environment for boolean speculations. In D. Skordev, editor, Mathematical logic and its applications, pages 253-263. Plenum Press, 1987.

[Hal95] Joseph Halpern. The effect of bounding the number of primitive propositions and the depth of nesting on the complexity of modal logic. Artificial Intelligence, 75(2):361-372, 1995.

[HC68] George Hughes and Max Cresswell. An introduction to modal logic. Methuen and Co., 1968.

[HM92] Joseph Halpern and Yoram Moses. A guide to completeness and complexity for modal logics of knowledge and belief. Artificial Intelligence, 54:319-379, 1992.

[Lad77] Richard Ladner. The computational complexity of provability in systems of modal propositional logic. SIAM Journal of Computing, 6(3):467-480, September 1977.

[Mas94] Fabio Massacci. Strongly analytic tableaux for normal modal logics. In Alan Bundy, editor, CADE-12, Nancy, pages 723-737. Springer Verlag, LNAI 814, July 1994.

[MMO95] P. Miglioli, U. Moscato, and M. Ornhagi. Refutation systems for propositional modal logics. In P. Baumgartner, Reiner Hähnle, and J. Posegga, editors, Workshop on Theorem Proving with Analytic Tableaux and Related Methods, pages 95-105. LNAI 918, Springer-Verlag, May 1995.

[Non95] Andreas Nonnengart. A resolution-based calculus for temporal logics. PhD thesis, Universität des Saarlandes, November 1995.

[Ogn94] Zoran Ognjanović. A tableau-like proof procedure for normal modal logics. Theoretical Computer Science, 129:167-186, 1994.

[OSH95] Hans Jürgen Ohlbach, R. Schmidt, and Hustadt. Translating graded modalities into predicate logic. Technical Report MPI-I-95-2-008, MaxPlanck-Institute Für Informatik, May 1995. 
[PT91] Solomon Passy and Tinko Tinchev. An essay in combinatory dynamic logic. Information and Computation, 93:263-332, 1991.

[Rij92] Maarten de Rijke. The modal logic of inequality. Journal of Symbolic Logic, 57(2):566-584, June 1992.

[Sat80] M. Sato. A cut-free Gentzen-type system for the modal logic S5. The Journal of Symbolic Logic, 45(1):67-84, March 1980.

[Seg81] Krister Segerberg. A note on the logic of elsewhere. Theoria, 47:183-187, 1981.

[Smu68] Raymond Smullyan. First-Order Logic. Springer-Verlag, 1968.

[WW85] Lincoln Wallen and G. Wilson. Automated deduction in S5 modal logic: theory and implementation. Technical Report DAI-315, University of Edinburgh, 1985. 